\title{
Post-Newtonian parameters and constraints on Einstein-aether theory
}

\author{
Brendan Z. Fostel* and Ted Jacobsont \\ Dept. of Physics, University of Maryland, \\ College Park, MD 20742-4111, USA
}

(Dated: February 7, 2006)

\begin{abstract}
We analyze the observational and theoretical constraints on "Einstein-aether theory", a generally covariant theory of gravity coupled to a dynamical, unit, timelike vector field that breaks local Lorentz symmetry. The results of a computation of the remaining post-Newtonian parameters are reported. These are combined with other results to determine the joint post-Newtonian, vacuumČerenkov, nucleosynthesis, stability, and positive-energy constraints. All of these constraints are satisfied by parameters in a large two-dimensional region in the four-dimensional parameter space defining the theory.
\end{abstract}

*bzf@umd.edu

ijacobson@umd.edu 
Alternative theories of gravity that deviate from general relativity have been ruled out or severely constrained systematically as observations have improved [1, 2]. At this stage the most studied surviving alternative is "scalar-tensor theory", of which Jordan-Brans-Dicke is a well-known example. This sort of theory is a simple extension of general relativity containing a fundamental scalar field. The existence of such scalar fields has been suggested by moduli fields that arise in higher dimensional approaches to gravity and quantum gravity, such as string theory.

Vector-tensor theories consisting of general relativity coupled to a dynamical vector field are much more complicated than scalar-tensor theories, due to the metric derivatives that appear in the covariant derivative of the vector field. They also appear to suffer from a glaring problem: because of the indefinite signature of the spacetime metric, some of the degrees of freedom are always associated with negative energies. This problem need not occur, however, if the vector field is constrained to have a fixed timelike magnitude. Such a vector field specifies a particular rest frame at each point of spacetime; hence, it "spontaneously" breaks the local Lorentz symmetry in a dynamical fashion. These theories - of which there is a four-parameter family - preserve the full diffeomorphism symmetry group of general relativity, and gravity is still described by the curvature of the spacetime metric. The existence of such a vector field is not as well-motivated as a scalar field, however a number of approaches to quantum gravity have very tentatively suggested that Lorentz symmetry might be broken. If general covariance is to be preserved, then any Lorentz violating vector (or tensor) field must be dynamical.

In this paper we discuss the complete collection of currently available observational and theoretical constraints on unit-vector-tensor theories, combining our new result for the remaining post-Newtonian parameters with previously established constraints. Surprisingly, all of these constraints are compatible with ranges of order unity for two coefficients in the Lagrangian. We are aware of no other theory that comes this close to so many predictions of general relativity and yet is fundamentally different.

We call the timelike unit vector the aether, and the coupled theory Einstein-aether theory, or ae-theory for short. A review of its properties together with a collection of references to earlier work was given in Ref. 3]. Ae-theory is defined by the action for the metric $g_{a b}$ 
and aether $u^{a}$. The most general action that is diffeomorphism-invariant and quadratic in derivatives is

$$
S=\frac{-1}{16 \pi G} \int d^{4} x \sqrt{-g}\left(R+K_{m n}^{a b} \nabla_{a} u^{m} \nabla_{b} u^{n}+\lambda\left(u^{a} u_{a}-1\right)\right)
$$

where

$$
K^{a b}{ }_{m n}=c_{1} g^{a b} g_{m n}+c_{2} \delta_{m}^{a} \delta_{n}^{b}+c_{3} \delta_{n}^{a} \delta_{m}^{b}+c_{4} u^{a} u^{b} g_{m n}
$$

The coefficients $c_{1,2,3,4}$ are dimensionless constants, $R$ is the Ricci scalar, and $\lambda$ is a Lagrange multiplier that enforces the unit constraint. The expression $R_{a b} u^{a} u^{b}$ is proportional to the difference of the $c_{2}$ and $c_{3}$ terms via integration by parts, hence is not an independent term. Note that since the covariant derivative of $u^{a}$ involves the Levi-Civita connection, which involves first derivatives of the metric, the aether part of the action in effect contributes also to the metric kinetic terms. We adopt the metric signature (+---), and units are chosen such that the speed of light defined by the metric $g_{a b}$ is unity. Other than the signature choice we use the conventions of Ref. [4].

Observations have already severely constrained Lorentz symmetry violation in the matter sector [5, 6], hence to a very good approximation matter must couple universally to one metric, which we take to be $g_{a b}$. Our goal is to determine the observational and theoretical constraints on the dimensionless parameters $c_{i}$.

In the weak-field, slow-motion limit ae-theory reduces to Newtonian gravity 7], with a value of Newton's constant $G_{\mathrm{N}}$ related to the constant $G$ in (11) by

$$
G_{\mathrm{N}}=G\left(1-\frac{c_{14}}{2}\right)^{-1}
$$

Here we have written $c_{14}$ for $c_{1}+c_{4}$, a notation we generalize below to $c_{123}=c_{1}+c_{2}+c_{3}$, etc.

A standard way of beginning to compare an alternative gravity theory to general relativity is to examine the first post-Newtonian corrections. For a general metric theory of gravity there are ten 'parametrized post-Newtonian' (PPN) parameters [1, 2] characterizing the lowest order effects in $v^{2} / c^{2}$ and dimensionless gravitational potential $G_{\mathrm{N}} M / c^{2} r$. Five of these parameters, $\zeta_{1}, \zeta_{2}, \zeta_{3}, \zeta_{4}$, and $\alpha_{3}$, vanish identically for any 'semi-conservative' theory, i.e. one derived, like ae-theory, from a covariant action principle. Two others, known as the Eddington-Robertson-Schiff parameters $\beta$ and $\gamma$, characterize respectively the nonlinearity 
and the spatial curvature produced by gravity. It was previously shown in Ref. [8] that in aetheory $\beta=\gamma=1$, just as in general relativity. Of the remaining three PPN parameters, two, $\alpha_{1}, \alpha_{2}$, characterize preferred frame effects, and the third, $\xi$ (sometimes called the Whitehead parameter), characterizes a peculiar sort of three-body interaction. The parameter $\alpha_{2}$ for ae-theory was computed in Ref. [9] to lowest nontrivial order in the parameters $c_{i}$.

Here we report on an ab initio computation of all the PPN parameters that confirms the previous results and determines the exact values of $\alpha_{2}$ and the previously undetermined parameters $\alpha_{1}$ and $\xi$. The parameters are defined by a weak-field expansion of the metric in terms of a set of generalized potentials defined by integrals over a fluid source. To determine the parameters, one solves the approximate field equations with the fluid source in a standard coordinate gauge. The computation is straightforward but lengthy, so the details are relegated to an appendix.

Our results indicate that the "time-time" and "space-space" components of the metric are the same in ae-theory and GR to calculated post-Newtonian order, where we refer to a nearly globally Lorentz coordinate system specialized to the standard PPN gauge and with the aether aligned with the time direction at zeroth-order. The "time-space" components of the metric $g_{0 i}, i=1,2,3$, differ as

$$
\left(g_{0 i}\right)_{\mathrm{ae}}-\left(g_{0 i}\right)_{\mathrm{GR}}=\frac{\alpha_{1}-\alpha_{2}}{2} V_{i}+\frac{\alpha_{2}}{2} W_{i}
$$

where $\alpha_{1,2}$ are the PPN parameters given explicitly below, while the components of the aether are

$$
\begin{gathered}
u^{0}=1+U \\
u^{i}=\frac{2 c_{1}+3 c_{2}+c_{3}+c_{4}}{2 c_{123}}\left(V_{i}-W_{i}\right)+\frac{\left(2-c_{14}\right)\left(c_{1}-c_{3}\right)}{2 c_{1}-c_{1}^{2}+c_{3}^{2}}\left(V_{i}+W_{i}\right) .
\end{gathered}
$$

The potentials $U, V_{i}$, and $W_{i}$ are defined by

$$
\left\{\begin{array}{c}
U(t, \mathbf{x}) \\
V_{i}(t, \mathbf{x}) \\
W_{i}(t, \mathbf{x})
\end{array}\right\}=G_{\mathrm{N}} \int d^{3} y \frac{\rho}{|\mathbf{x}-\mathbf{y}|}\left\{\begin{array}{c}
1 \\
v^{i} \\
\frac{v^{j}\left(x^{j}-y^{j}\right)\left(x^{i}-y^{i}\right)}{|\mathbf{x}-\mathbf{y}|^{2}}
\end{array}\right\},
$$

where $\rho(t, \mathbf{y})$ is the rest-mass energy density of the fluid source, $v^{\mu}(t, \mathbf{y})$ is the fluid fourvelocity, and the repeated indices are summed over. 
The components of the perturbed metric show that the ae-theory PPN parameters are given by

$$
\begin{gathered}
\gamma=\beta=1 \\
\xi=\zeta_{1}=\zeta_{2}=\zeta_{3}=\zeta_{4}=\alpha_{3}=0 \\
\alpha_{1}=\frac{-8\left(c_{3}^{2}+c_{1} c_{4}\right)}{2 c_{1}-c_{1}^{2}+c_{3}^{2}} \\
\alpha_{2}=\frac{\left(2 c_{13}-c_{14}\right)^{2}}{c_{123}\left(2-c_{14}\right)} \\
-\frac{12 c_{3} c_{13}+2 c_{1} c_{14}\left(1-2 c_{14}\right)+\left(c_{1}^{2}-c_{3}^{2}\right)\left(4-6 c_{13}+7 c_{14}\right)}{\left(2-c_{14}\right)\left(2 c_{1}-c_{1}^{2}+c_{3}^{2}\right)} .
\end{gathered}
$$

Note that the parameters $\alpha_{1}$ and $\alpha_{2}$ are both of linear order in $c_{i}$ when the coefficients are small compared to unity and the ratios amongst them are of order unity.

It is evident from the form of the metric and aether perturbations that the cases $c_{123}=0$, $c_{14}=2$, and $2 c_{1}-c_{1}^{2}+c_{3}^{2}=0$ are special, since $\alpha_{1}$ and/or $\alpha_{2}$ diverges. Presumably the post-Newtonian approximation is not valid when the coefficients are close to these values. From the wave speeds (15) below we see that the spin-0 speed vanishes in either of the first two cases and the spin- 1 speed vanishes in the last case. This corresponds to the absence of spatial gradient terms in the action [9]. The case $c_{123}=0$ corresponds [8] to the vector-tensor theory of Hellings and Nordtvedt [10] if the unit constraint on the aether is dropped. This theory was shown by Will [1] to be dynamically over-determined and hence observationally unacceptable.

The current best constraints [1, 2] on the preferred frame PPN parameters are $\left|\alpha_{1}\right| \lesssim 10^{-4}$ (from an orbital polarization effect bounded by lunar laser ranging and binary pulsar observations) and $\left|\alpha_{2}\right| \lesssim 4 \times 10^{-7}$ (from a spin precession effect bounded by the alignment of the solar spin with the ecliptic). These two conditions can be met with two unrestricted parameters to spare, since we begin with four free parameters $c_{1,2,3,4}$. At the lowest resolution, we can just impose $\alpha_{1}=\alpha_{2}=0$. The condition $\alpha_{1}=0$ implies $c_{4}=-c_{3}^{2} / c_{1}$. (The case $c_{1}=0$ can be excluded since it yields $\alpha_{1}=-8$, which lies far outside the observational bound.) Having put $\alpha_{1}=0$ in this way, it can be seen (the seeing made easier with the help of Mathematica) that $\alpha_{2}$ can be put to zero in two ways. One way is with $c_{13}=0$, which together with the previous condition implies also $c_{14}=0$. This case is degenerate, and is briefly discussed at the end of this paper. The other way to put both $\alpha_{1}$ and $\alpha_{2}$ to zero is 
to determine $c_{2}$ and $c_{4}$ in terms of $c_{1}$ and $c_{3}$ via

$$
\begin{aligned}
& c_{2}=\left(-2 c_{1}^{2}-c_{1} c_{3}+c_{3}^{2}\right) / 3 c_{1} \\
& c_{4}=-c_{3}^{2} / c_{1}
\end{aligned}
$$

Thus there is a two-parameter family of ae-theory Lagrangians for which all the PPN parameters are identical to those of $G R$.

We now consider the other constraints on ae-theory. In alternate gravity theories including Brans-Dicke theory, the Newton constant $G_{\mathrm{N}}$ need not be constant in time. Observational bounds on $\dot{G} / G$ then constrain the theory. In the case of ae-theory, we have the relation (3), hence $G_{\mathrm{N}}$ is always constant.

Another constraint arises from the possible discrepancy between Newton's constant and the gravitational constant occurring in the equation for the dynamics of the cosmological scale factor. In general relativity, the scale factor satisfies the Friedman equation, which involves Newton's constant. In ae-theory, when the metric has the standard cosmological form (Robertson-Walker symmetry) and the aether is aligned with the cosmological rest frame, the aether stress tensor can be constructed purely from the spacetime metric with two derivatives, and must be identically divergence free. It must therefore be a linear combination of the Einstein tensor $G_{a b}$ and a tensor constructed with the spatial curvature scalar ${ }^{(3)} R$, which turns out to be [7, 11]:

$$
T_{a b}^{\text {aether }}=-\frac{c_{13}+3 c_{2}}{2}\left[G_{a b}-\frac{1}{6}{ }^{(3)} R\left(g_{a b}+2 u_{a} u_{b}\right)\right] .
$$

The effect of the cosmological aether is thus to renormalize the gravitational constant and to add a stress tensor of perfect fluid type that in effect renormalizes the spatial curvature contribution to the field equations. The renormalized, cosmological gravitational constant is given by [7]

$$
G_{\text {cosmo }}=G\left(1+\frac{c_{13}+3 c_{2}}{2}\right)^{-1}
$$

Since this is not the same as $G_{\mathrm{N}}(\underline{3})$, the expansion rate of the universe differs from what would have been expected in GR with the same matter content. The ratio is constrained by the observed primordial ${ }^{4} \mathrm{He}$ abundance to satisfy $\left|G_{\text {cosmo }} / G_{\mathrm{N}}-1\right|<1 / 8$, which imposes a constraint on the constants $c_{i}$ [7]. Remarkably, if the constants are restricted by (12) so that $\alpha_{1,2}=0$, then $G_{\mathrm{N}}=G_{\text {cosmo. }}$. Primordial nucleosynthesis then imposes no additional constraint. 
Even when the two gravitational constants coincide, the "curvature fluid" term in (13) represents a deviation from the Friedman equation in GR if the universe has non-zero spatial curvature. Observations have shown that the spatial curvature must be very small today, and it would have been even less important in the past when the relative contribution of matter and radiation would have been even more important. It thus seems unlikely that an interesting constraint can be obtained from this term. Another potential source of cosmological constraint is the modification of the primordial fluctuation spectrum [12], but this has not yet been worked out in full detail.

A further constraint on ae-theory comes from the possibility that the gravity and aether waves travel at less than the speed of "light" - that is, less than the limiting speed determined by the metric $g_{a b}$ governing the propagation of matter fields. In this case, high energy matter moving inertially through the vacuum would produce vacuum Cerenkov radiation of gravitational and aether shock waves. A detailed analysis of this process and the corresponding observational constraints from ultra-high-energy cosmic ray observations was carried out in Ref. [13]. The constraints are characterized by very small numbers, ranging between $10^{-15}$ and $10^{-31}$, depending on the wave-mode type and emission process. These are all one-sided constraints, since they apply only when the wave speeds are smaller than the speed of light. To a first approximation then, the constraints imply that the wave speeds must be greater than the speed of light.

Some authors [7, 12, 13] have suggested that superluminal propagation be excluded $a$ priori on the grounds that ae-theory should be viewed as an effective description of an underlying Lorentz invariant theory in a configuration with broken Lorentz symmetry. However, this is a purely theoretical bias, with no observational basis that we can see. Moreover, superluminal propagation does not threaten causality, as long as there is a limiting speed in at least one given reference frame, as there is in ae-theory. We thus adopt a phenomenological stance, allowing for superluminal propagation unless-and-until it is observationally ruled out.

There are five gravitational and aether wave modes in ae-theory: two correspond to the usual gravitational spin-2 waves, two are a transverse spin-1 aether-gravity wave, and one is a longitudinal spin-0 aether-gravity wave. The squared speeds of these modes are determined 
by the constants $c_{i}$, and are given by 14

$$
\begin{array}{ll}
\text { spin-2 } & 1 /\left(1-c_{13}\right) \\
\text { spin-1 } & \left(c_{1}-\frac{1}{2} c_{1}^{2}+\frac{1}{2} c_{3}^{2}\right) / c_{14}\left(1-c_{13}\right) \\
\text { spin-0 } & c_{123}\left(2-c_{14}\right) / c_{14}\left(1-c_{13}\right)\left(2+c_{13}+3 c_{2}\right) .
\end{array}
$$

If we impose the $\alpha_{1,2}=0$ conditions (12), the Cerenkov constraint that the spin-2 and spin-0 wave speeds be superluminal restricts $c_{1}$ and $c_{3}$ to the region

$$
\begin{aligned}
& 0<c_{13}<1 \\
& 0<\left(c_{1}-c_{3}\right)<c_{13} / 3\left(1-c_{13}\right) .
\end{aligned}
$$

These conditions also ensure that the spin-1 wave speed is superluminal.

In addition to the observational constraints already mentioned, there are two theoretical constraints, coming from the requirement that the wave modes be stable-i.e. have real frequencies - and that the energy of the modes be positive. The first requirement is already guaranteed by the no-Čerenkov condition that the speeds be greater than unity. The signs of the energy densities of the wave modes, averaged over a cycle, are given by [15]

$$
\begin{array}{ll}
\text { spin-2 } & 1 \\
\text { spin-1 } & \left(2 c_{1}-c_{1}^{2}+c_{3}^{2}\right) /\left(1-c_{13}\right) \\
\text { spin-0 } & c_{14}\left(2-c_{14}\right) .
\end{array}
$$

The spin-2 modes always carry positive energy. If the $\alpha_{1,2}=0$ conditions (12) and the superluminal conditions (16) are satisfied, then we find that the spin-1 and spin-0 modes also carry positive energy. By contrast, if the speeds are sub-luminal, then the latter two modes carry negative energy. Thus, not only the Cerenkov constraints, but also the positive energy requirement excludes the case of sub-luminal wave speeds.

We earlier pointed out that an alternate way to set $\alpha_{1}=\alpha_{2}=0$ is if $c_{13}=c_{14}=0$. In this case $G_{\mathrm{N}} / G_{\text {cosmo }}=\left(1+3 c_{2} / 2\right)$, so nucleosynthesis would impose a constraint on $c_{2}$. A more serious concern is that the spin-0 and spin-1 wave speeds (15) diverge in this case, because there are no time derivative terms in the aether field equation [14]. At the same time the energy density (17) of the spin-0 mode goes to zero, but that of the spin-1 mode remains finite. We have not worked out the observational signatures of this behavior.

It is rather non-trivial that the PPN parameters are identical to those of GR and that the vacuum Čerenkov, nucleosynthesis, stability, and positive energy constraints are all satisfied 
in a large two-dimensional region (12 16) in the four-dimensional $c_{i}$ parameter space. To further constrain the parameters one should look to strong-field effects or radiative processes. Since the gravitational radiation damping of binary pulsars has been found to agree with GR to one part in $10^{3}$, one would expect constraints of order $10^{-3}$ from such observations. The ae-theory radiation damping rate has been determined to lowest non-trivial order [16], but higher-order terms are still needed to set definitive constraints.

One particular strong-field effect that can be examined is the existence and nature of black hole solutions to the vacuum field equations. Some alternate theories of gravity whose PPN parameters are equal or close to those of GR do not admit regular black hole solutions. In these theories, astrophysical collapse would produce something other than a black hole - perhaps a naked singularity, or a bounce - which may not be difficult to rule out ob-

servationally. Recent studies [17] have shown, however, that ae-theory does admit regular black hole solutions.

In conclusion, ae-theory provides a healthy sparring partner for GR, a role that was previously played only by scalar-tensor theory. One of the motivations for exploring this theory is the idea that the existence of a preferred timelike direction in spacetime could play a role in the solution to fundamental problems such as the nature of dark energy or quantum gravity. Although it is likely that radiation and strong-field constraints will limit all of the theory's parameters to be small compared to unity, they would still allow the aether field to exist and possibly play such a role.

\section{Acknowledgments}

We thank Konstantin Zloshchastiev for noting several typos in an earlier draft of the appendix. This research was supported in part by the NSF under grant PHY-0300710 at the University of Maryland.

\section{APPENDIX: CALCULATION OF AE-THEORY PPN PARAMETERS}

This appendix provides details of the calculation of the Parameterized Post-Newtonian (PPN) parameters, $\alpha_{1}, \alpha_{2}, \alpha_{3}, \beta, \gamma, \zeta_{1}, \zeta_{2}, \zeta_{3}, \zeta_{4}, \xi$, for ae-theory. The PPN formalism is defined in a weak-field, slow-motion limit, and describes the next-to-Newtonian order gravitational 
effects in terms of a standardized set of potentials and these ten parameters. We will determine the PPN parameters by solving the field equations with a perfect-fluid source in a standard coordinate gauge order-by-post-Newtonian-order. More detailed explanations of the procedure and the general PPN formalism can be found in the classic reference of Will [1].

We will follow the conventions of [4], with the following exceptions and additions. The metric will have signature $(+,-,-,-)$. We will write the approximate equations in terms of components of tensors with all indices lowered. Spatial indices will be indicated by lowercase Latin letters from the middle of the alphabet: $i, j, k, \ldots$. Spatial indices are raised and lowered with the spacetime metric; e.g. $v_{i} \equiv v^{a} g_{a i}=-v^{i}+$ (higher order terms). Repeated spatial indices are summed over. We will write, e.g. $c_{14}$ for $c_{1}+c_{4}$, etc. Note also that we follow modern conventions for denoting the post-Newtonian order of quantities (see, e.g. [2]), which differ from the conventions of [1].

The ae-theory field equations follow from the action (1), with an additional perfect-fluid source coupled in the standard way to the metric $g_{a b}$, and uncoupled to the aether $u^{a}$. We have the Einstein equations, written in the non-standard form

$$
R_{a b}=\left(S_{c d}+8 \pi G T_{c d}\right)\left(\delta_{a}^{c} \delta_{b}^{d}-\frac{1}{2} g_{a b} g^{c d}\right)
$$

where

$$
\begin{aligned}
S_{a b}= & \nabla_{m}\left(J_{(a}^{m} u_{b)}-J_{(a}^{m} u_{b)}-J_{(a b)} u^{m}\right) \\
& +c_{1}\left(\nabla_{m} u_{a} \nabla^{m} u_{b}-\nabla_{a} u_{m} \nabla_{b} u^{m}\right)+c_{4} \dot{u}_{a} \dot{u}_{b} \\
& +\lambda u_{a} u_{b}+\frac{1}{2} g_{a b}\left(J_{m}^{n} \nabla_{n} u^{m}\right) ;
\end{aligned}
$$

with

$$
J_{m}^{a}=\left(c_{1} g^{a b} g_{m n}+c_{2} \delta_{m}^{a} \delta_{n}^{b}+c_{3} \delta_{n}^{a} \delta_{m}^{b}+c_{4} u^{a} u^{b} g_{m n}\right) \nabla_{b} u^{n}
$$

and

$$
\dot{u}^{a}=u^{b} \nabla_{b} u^{a}
$$

also

$$
T^{a b}=(\rho+\rho \Pi+p) v^{a} v^{b}-p g^{a b},
$$

where $v^{a}$ is the four-velocity, $\rho$ the rest-mass-energy density, $\Pi$ the internal energy density, and $p$ the isotropic pressure of the fluid. We also have the aether field equation

$$
\nabla_{a} J_{m}^{a}-c_{4} \dot{u}_{a} \nabla_{m} u^{a}=\lambda u_{m}
$$


and the constraint

$$
g_{a b} u^{a} u^{b}=1 .
$$

Eqn. (A.6) can be used to eliminate $\lambda$, giving

$$
\lambda=u^{m} \nabla_{a} J_{m}^{a}-c_{4} \dot{u}^{a} \dot{u}_{a} .
$$

We assume a nearly-globally-Lorentzian coordinate system and basis with respect to which, at zeroth order, the metric is the Minkowski metric $\eta_{a b}$ and the aether is purely timelike. The fluid variables are assigned orders of $\rho \sim \Pi \sim p / \rho \sim\left(v^{i}\right)^{2} \sim O(1)$. Taking the time-derivative of a quantity will effectively raise its order by one-half: $X \sim O(N) \rightarrow$ $\partial X / \partial t \sim O(N+1 / 2)$. We assume that the components of the metric perturbations $h_{a b}$ with respect to this basis will be of orders

$$
h_{00} \sim O(1)+O(2), \quad h_{i j} \sim O(1), \quad h_{0 i} \sim O(1.5) .
$$

This assignment preserves the Newtonian limit while allowing one to determine just the first post-Newtonian corrections. The aether perturbations $\delta u^{a}$ are assumed to be of orders

$$
\delta u^{0} \sim O(1), \quad \delta u^{i} \sim O(1.5) .
$$

Lower orders are disallowed by the field equations, given the above orders of $h_{a b}$. We will assume that $h_{a b}$ and $\delta u^{a}$ satisfy boundary conditions such that they vanish at spatial infinity.

The metric components are to be expanded in terms of particular potential functions, thus defining the PPN parameters:

$$
\begin{aligned}
g_{00}= & 1-2 U+2 \beta U^{2}+2 \xi \Phi_{W}-\left(2 \gamma+2+\alpha_{3}+\zeta_{1}-2 \xi\right) \Phi_{1} \\
& -2\left(3 \gamma-2 \beta+1+\zeta_{2}+\xi\right) \Phi_{2}-2\left(1+\zeta_{3}\right) \Phi_{3}-2\left(3 \gamma+3 \zeta_{4}-2 \xi\right) \Phi_{4}+\left(\zeta_{1}-2 \zeta\right) \mathcal{A}, \\
g_{i j}= & -(1+2 \gamma U) \delta_{i j}, \\
g_{0 i}= & \frac{1}{2}\left(4 \gamma+3+\alpha_{1}-\alpha_{2}+\zeta_{1}-2 \xi\right) V_{i}+\frac{1}{2}\left(1+\alpha_{2}+\zeta_{1}+2 \xi\right) W_{i} .
\end{aligned}
$$

The potentials are all of the form

$$
F(x)=G_{\mathrm{N}} \int d^{3} y \frac{\rho(y) f}{|x-y|},
$$


where $G_{\mathrm{N}}$ is the current value of Newton's constant, which we determine below in terms of $G$ and the $c_{i}$. The correspondences $F: f$ are given by

$$
\begin{gathered}
U: 1 \quad \Phi_{1}: v_{i} v_{i} \quad \Phi_{2}: U \quad \Phi_{3}: \Pi \quad \Phi_{4}: p / \rho \\
\Phi_{W}: \int d^{3} z \rho(z) \frac{(x-y)_{j}}{|x-y|^{2}}\left(\frac{(y-z)_{j}}{|x-z|}-\frac{(x-z)_{j}}{|y-z|}\right) \quad \mathcal{A}: \frac{\left(v_{i}(x-y)_{i}\right)^{2}}{|x-y|^{2}} \\
V_{i}: v^{i} \quad W_{i}: \frac{v_{j}\left(x_{j}-y_{j}\right)\left(x^{i}-y^{i}\right)}{|x-y|^{2}} .
\end{gathered}
$$

Note that for $U, \Phi_{1,2,3,4}$, and $V_{i}$,

$$
F_{, i i}=-4 \pi G_{\mathrm{N}} \rho f
$$

We will also make use of the 'superpotential' $\chi$ :

$$
\chi=-G_{\mathrm{N}} \int d^{3} y \rho|x-y|
$$

which satisfies

$$
\chi_{, i i}=-2 U
$$

We also note the relation

$$
\chi_{, i 0}=V_{i}-W_{i},
$$

which follows from the formula

$$
\frac{\partial}{\partial t} \int d^{3} y \rho(\mathbf{y}, t) f(\mathbf{x}, \mathbf{y})=\int d^{3} y \rho(\mathbf{y}, t) v^{i}(\mathbf{y}, t) \frac{\partial f}{\partial y^{i}}[1+O(1)]
$$

which follows from the continuity equation for the fluid

$$
\rho_{, 0}+\left(\rho v^{i}\right)_{, i}=0
$$

assumed to hold to $O(1.5)$.

These potentials satisfy certain criteria of "reasonableness" and simplicity (see [1], Sec. (4.1) for details), and are general enough to describe all known viable theories of gravity. In particular, they suffice for ae-theory. The criteria permit $g_{00}$ to depend also on the potential $\chi_{, 00}$, and $g_{i j}$ to depend on $\chi_{, i j}$. Such terms, however, can always be eliminated [1] by a suitable coordinate transformation that preserves the zeroth-order form of the components. The 'standard PPN gauge' is thus defined as that post-Newtonian coordinate frame in which 
all dependence on $\chi_{, 00}$ and $\chi_{, i j}$ has been removed from, respectively, $g_{00}$ and $g_{i j}$. This fixing determines the coordinate frame up to necessary order so that the standard form of the metric components is unambiguous.

In carrying out the calculations, we shall impose the following gauge conditions:

$$
\begin{gathered}
h_{i j, j}=-\frac{1}{2}\left(h_{00, i}-h_{j j, i}\right) \\
h_{0 i, i}=-3 U_{,_{0}}+\theta n_{i, i}
\end{gathered}
$$

where $\theta$ is an arbitrary parameter and $n_{i}=u_{i}-h_{0 i}$. These conditions are suggested by the standard conditions for general relativity. As we shall see, the conditions (A.20) suffice to put $g_{i j}$ in standard form, while the fourth condition (A.21) standardizes $g_{00}$ when

$$
\theta=-\frac{\left(c_{1}+2 c_{3}-c_{4}\right)}{\left(2-c_{14}\right)}
$$

The solving procedure is as follows:

Step 1: Solve the constraint A.7. for $u^{0}$ to $O(1)$;

Step 2: Solve the "time-time" component of the Einstein equation (A.1) for $g_{00}$ to $O(1)$

Step 3: Solve the "space-space" components of (A.1) for $g_{i j}$ to $O(1)$;

Step 4: Solve the "space" components of the aether field equation (A.6) for $u^{i}$ to $O(1.5)$;

Step 5: Solve the "time-space" components of (A.1) for $g_{0 i}$ to $O(1.5)$;

Step 6: Solve the "time-time" component of (A.1) for $g_{00}$ to $O(2)$.

The cases in which $c_{123}=0, c_{14}=2$, or $2 c_{1}-c_{1}^{2}+c_{3}^{2}=0$ are special in that the found solutions diverge. We will presume that the post-Newtonian approximation is not valid in these cases, and assume below that they do not hold. See the main text for more discussion of this point.

\section{1. $u^{0}$ to $O(1)$}

Solving the constraint (A.7) gives

$$
u^{0}=1-(1 / 2) h_{00}
$$


to $O(1)$. For the components of $u_{a}$, we have

$$
u_{0} \equiv u^{a} g_{a 0}=1+\frac{1}{2} h_{00}
$$

and

$$
u_{i}=u^{a} g_{a i}=-u^{i}+h_{0 i}=n_{i}+h_{0 i}
$$

For later convenience, we will now express the covariant derivatives of $u_{a}$. The constraint (A.7) implies that

$$
\nabla_{a} u_{0}=0
$$

to $O(2)$. Also to $O(2)$, we have

$$
\nabla_{0} u_{i}=-\frac{1}{2} h_{00, i}\left(1-\frac{1}{2} h_{00}\right)+h_{0 i, 0}+n_{i, 0}
$$

and

$$
\dot{u}_{i}=u^{0} \nabla_{0} u_{i}=-\frac{1}{2} h_{00, i}\left(1-h_{00}\right)+h_{0 i, 0}+n_{i, 0} .
$$

To $O(1.5)$, we have

$$
\nabla_{j} u_{i}=n_{i, j}+\frac{1}{2} h_{i j, 0}+h_{0[i, j]}
$$

\section{2. $g_{00}$ to $O(1)$}

We now solve the "time-time" component of the Einstein equation (A.1) for $g_{00}$ to $O(1)$. For the components of $R_{00}$, we have

$$
R_{00}=\frac{1}{2} h_{00, i i}+\frac{1}{2} h_{i j} h_{00, i j}-\left(h_{i 0, i}-\frac{1}{2} h_{i i, 0}\right)_{, 0}-\frac{1}{4} h_{00, i} h_{00, i}+\frac{1}{4} h_{00, j}\left(2 h_{i j, i}-h_{i i, j}\right)
$$

to $O(2)$. At $O(1)$, we have

$$
\begin{gathered}
R_{00}=\frac{1}{2} h_{00, i i}, \\
T_{00}=\rho, \quad T_{i j}=0, \\
S_{00}=J_{0}{ }^{m}{ }_{, m}-J_{00,0}=-J_{0 i, i}=-c_{14}\left(\nabla_{0} u_{i}\right)_{, i}=\frac{c_{14}}{2} h_{00, i i}, \\
S_{i j}=0 .
\end{gathered}
$$

The field equation becomes

$$
\left(1-\frac{c_{14}}{2}\right) h_{00, i i}=8 \pi G \rho,
$$


which gives $h_{00}$ to $\mathrm{O}(1)$,

$$
h_{00}=-2 U
$$

with Newton's constant

$$
G_{N}=\left(1-\frac{c_{14}}{2}\right)^{-1} G
$$

\section{3. $g_{i j}$ to $O(1)$}

We now solve the "space-space" components of A.1 for $g_{i j}$ to $O(1)$. We have to $O(1)$

$$
\begin{aligned}
R_{i j} & =\frac{1}{2} h_{i j, k k}+\frac{1}{2} h_{k k, i j}-h_{k(i, j) k}-\frac{1}{2} h_{00, i j} \\
& =\frac{1}{2} h_{i j, k k},
\end{aligned}
$$

where we have imposed the gauge condition (A.20) in the second step. Using (A.32), (A.33), and (A.34), the field equation becomes

$$
h_{i j, k k}=8 \pi G_{N} \rho \delta_{i j}
$$

giving

$$
h_{i j}=-2 U \delta_{i j}
$$

\section{4. $u^{i}$ to $O(1.5)$}

We now solve the "space" components of the aether field equation (A.6) for $u^{i}$ to $O(1.5)$, making use of the gauge condition (A.20) and our earlier results (A.24), (A.36), and (A.40). At $O(1.5)$ equation (A.6) has the form

$$
J_{i, a}^{a}=J_{0 i, 0}-J_{j i, j}=0
$$

To $O(1.5)$

$$
J_{0 i, 0}=c_{14}\left(\nabla_{0} u_{i}\right)_{, 0}=-\frac{c_{14}}{2} h_{00, i 0}=-\frac{c_{14}}{2} \chi_{, 0 i j j}
$$

and

$$
\begin{aligned}
J_{j i, j} & =\left(c_{1} \nabla_{j} u_{i}+c_{2} \delta_{i j} \nabla_{k} u_{k}+c_{3} \nabla_{i} u_{j}\right) \\
& =c_{1} n_{i, j j}+c_{23} n_{j, j i}+\frac{1}{2}\left(2 c_{-} h_{0[i, j] j}+\left(c_{+}+3 c_{2}\right) \chi_{, 0 i j j}\right),
\end{aligned}
$$


where $c_{-}=c_{1}-c_{3}, c_{+}=c_{13}$. The aether field equation can then be written

$$
\left(c_{1} n_{i}+\frac{c_{-}}{2} h_{0 i}+\frac{1}{2}\left(2 c_{1}+3 c_{2}+c_{3}+c_{4}\right) \chi_{, i 0}\right)_{, j j}-\left(\frac{c_{-}}{2} h_{0 j, j}-c_{23} n_{j, j}\right)_{, i}=0 .
$$

Taking the spatial divergence of the left-hand side gives the relation

$$
n_{i, i j j}=A \chi_{, 0 i i j j}
$$

where

$$
A=-\frac{2 c_{1}+3 c_{2}+c_{3}+c_{4}}{2 c_{123}},
$$

which we can solve for $n_{i, i}$. Substituting into (A.44), imposing the gauge condition (A.21), and using our earlier results, we solve (A.44):

$$
n_{i}=-u^{i}=-\frac{1}{2 c_{1}}\left(c_{-} h_{0 i}-\left(2 c_{1} A+c_{-}\left(\frac{3}{2}+A \theta\right)\right) \chi_{, 0 i}\right) .
$$

\section{5. $g_{0 i}$ to $O(1.5)$}

We now solve the "time-space" components of (A.1) for $g_{0 i}$ to $O(1.5)$, making use of the gauge conditions (A.20) and (A.21) and the earlier results (A.24), (A.36), (A.40), and (A.47). We have to $O(1.5)$

$$
\begin{aligned}
R_{0 i} & =h_{0[i, j] j}+h_{j[j, i] 0} \\
& =\frac{1}{2}\left(h_{0 i}+\frac{1}{2}(1-2 A \theta) \chi_{, 0 i}\right)_{, j j},
\end{aligned}
$$

Also to $O(1.5)$,

$$
T_{0 i}=\rho v_{i},
$$

and

$$
S_{0 i}=-J_{(i 0), 0}+\frac{1}{2} J_{i}{ }^{m}{ }_{, m}=-\frac{1}{2}\left(J_{0 i, 0}+J_{i j, j}\right) .
$$

We have

$$
\begin{aligned}
J_{i j, j} & =\left(c_{1} \nabla_{i} u_{j}+c_{2} \delta_{i j} \nabla_{k} u_{k}+c_{3} \nabla_{j} u_{i}\right)_{, j} \\
& =\left(c_{12} n_{j, i}+c_{3} n_{i, j}\right)_{, j}+\frac{1}{2}\left(2 c_{-} h_{0[j, i] j}+c_{+} h_{i j, j 0}+c_{2} h_{j j, i 0}\right) \\
& =\left(-\frac{c_{-} c_{+}}{2 c_{1}} h_{0 i}+\left(\frac{c_{14}}{2}-E\right) \chi_{, 0 i}\right)_{, j j},
\end{aligned}
$$

where

$$
E=\frac{1}{4 c_{1}}\left(c_{1}^{2}+3 c_{3}^{2}+4 c_{1} c_{4}-2 c_{-} c_{+} A \theta\right) .
$$


With (A.42), this gives

$$
S_{0 i}=\left(\frac{c_{-} c_{+}}{4 c_{1}} h_{0 i}+\frac{E}{2} \chi_{, 0 i}\right)_{, j j} .
$$

The field equation becomes

$$
\left(1-\frac{c_{-} c_{+}}{2 c_{1}}\right) h_{0 i, j j}=16 \pi G \rho v_{i}+\left(E+A \theta-\frac{1}{2}\right) \chi_{, 0 i j j},
$$

giving

$$
h_{0 i}=\left(1-\frac{c_{-} c_{+}}{2 c_{1}}\right)^{-1}\left(\left(E+A \theta-\frac{1}{2}\right) \chi_{, 0 i}+4\left(1-\frac{c_{14}}{2}\right) V_{i}\right) .
$$

\section{6. $\quad g_{00}$ to $O(2)$}

We now solve the "time-time" component of (A.1) for $g_{00}$ to $O(2)$, making use of the gauge conditions (A.20) and (A.21) and the earlier results (A.24), (A.36), A.40, (A.47), and (A.55). Define $\tilde{h}_{00}=g_{00}-1+2 U$. Then, from eqn. (A.30), we have

$$
R_{00}=\frac{1}{2}\left(\tilde{h}_{00}-2 U-2 U^{2}+8 \Phi_{2}-2 A \theta \chi_{, 00}\right)_{, i i} .
$$

Also,

$$
\begin{gathered}
T_{00}=\rho\left(1+\Pi+v_{i} v_{i}-2 U\right), \\
T_{i j}=\rho v_{i} v_{j}+p \delta_{i j} . \\
g_{00}\left(T_{a b} g^{a b}\right)=(1-2 U)\left(T^{00}(1-2 U)-T^{i i}(1+2 U)\right),
\end{gathered}
$$

so that

$$
\begin{aligned}
T_{00}-\frac{1}{2} g_{00}\left(T_{a b} g^{a b}\right) & =\frac{1}{2} \rho\left(1+\Pi+2\left(v_{i} v_{i}-U\right)\right)+\frac{3}{2} p \\
& =\frac{-\left(1-c_{14} / 2\right)}{8 \pi G}\left(U+2 \Phi_{1}-2 \Phi_{2}+\Phi_{3}+3 \Phi_{4}\right)_{, i i} .
\end{aligned}
$$

To aid the reader in sorting through the terms appearing in $S_{a b}$, we note that to $O(2)$, in the chosen gauge,

$$
\nabla_{i} u_{i}=\left(\frac{3}{2}+A\right) \chi_{, 0 i i}
$$

and

$$
\left(\nabla_{0} u_{i}\right)_{, i}=-\frac{1}{2}\left(-2 U+\tilde{h}_{00}-U^{2}-2\left(\frac{3}{2}+(1+\theta) A\right) \chi_{, 00}\right) .
$$


After some bookkeeping, we find that

$$
S_{00}=\frac{c_{14}}{2}\left(-2 U+\tilde{h}_{00}-\frac{5}{2} U^{2}+9 \Phi_{2}\right)_{, i i}-c_{14}\left(\frac{3}{2}+(1+\theta) A\right) \chi_{, 00 i i},
$$

and

$$
S_{i i}=\frac{c_{14}}{2}\left(\frac{1}{2} U^{2}-\Phi_{2}\right)_{, i i}-\left(c_{+}+3 c_{2}\right)\left(\frac{3}{2}+A\right) \chi_{, 00 i i}
$$

We thus have

$$
\begin{aligned}
S_{00}-\frac{1}{2} g_{00} S_{a b} g^{a b}= & \frac{1}{2}\left(S_{00}+S_{i i}\right)+2 U S_{i i} \\
= & \frac{c_{14}}{4}\left(-2 U+\tilde{h}_{00}-2 U^{2}+8 \Phi_{2}\right)_{, i i} \\
& -\frac{1}{2}\left(\left(\frac{3}{2}+A\right)\left(2 c_{1}+3 c_{2}+c_{3}+c_{4}\right)+c_{14} A \theta\right) \chi_{, 00 i i} .
\end{aligned}
$$

We combine (A.56), (A.60), and (A.65), solve the field equation, and obtain

$$
\tilde{h}_{00}=2 U^{2}-4 \Phi_{1}-4 \Phi_{2}-2 \Phi_{3}-6 \Phi_{4}+Q \chi, 00
$$

where

$$
Q=\left(1-\frac{c_{14}}{2}\right)^{-1}\left(\left(2-c_{14}\right) \theta+\left(c_{1}+2 c_{3}-c_{4}\right)\right) A .
$$

Finally, we move into the standard gauge by choosing $\theta$ so that $Q$ vanishes:

$$
\theta \longrightarrow \theta_{0}=-\frac{c_{1}+2 c_{3}-c_{4}}{2-c_{14}}
$$

\section{Summary}

We now collect our results, eqns. (A.36), (A.40), (A.55), and (A.66) for the metric components, and (A.23) and (A.47) for the aether, imposing the gauge conditions (A.20) and (A.21) with $\theta=\theta_{0}$, and using the relation (A.17). For the metric, we have

$$
\begin{aligned}
& g_{00}=1-2 U+2 U^{2}-4 \Phi_{1}-4 \Phi_{2}-2 \Phi_{3}-6 \Phi_{4} \\
& g_{i j}=-(1+2 U) \delta_{i j} \\
& g_{0 i}=\frac{2 c_{1}}{2 c_{1}-c_{1}^{2}+c_{3}^{2}}\left(\left(E+A \theta_{0}-\frac{1}{2}+2\left(2-c_{14}\right)\right) V_{i}-\left(E+A \theta_{0}-\frac{1}{2}\right) W_{i}\right) .
\end{aligned}
$$


We can extract the PPN parameters by comparison with the standard forms (A.11). We find

$$
\begin{gathered}
\gamma=\beta=1 \\
\xi=\zeta_{1}=\zeta_{2}=\zeta_{3}=\zeta_{4}=\alpha_{3}=0 \\
\alpha_{1}=-\frac{8\left(c_{3}^{2}+c_{1} c_{4}\right)}{2 c_{1}-c_{1}^{2}+c_{3}^{2}} \\
\alpha_{2}=\frac{\left(2 c_{13}-c_{14}\right)^{2}}{c_{123}\left(2-c_{14}\right)} \\
-\frac{12 c_{3} c_{13}+2 c_{1} c_{14}\left(1-2 c_{14}\right)+\left(c_{1}^{2}-c_{3}^{2}\right)\left(4-6 c_{13}+7 c_{14}\right)}{\left(2-c_{14}\right)\left(2 c_{1}-c_{1}^{2}+c_{3}^{2}\right)}
\end{gathered}
$$

For the aether, we have

$$
\begin{aligned}
& u^{0}=1+U \\
& u^{i}=-(A+B) V_{i}+(A-B) W_{i},
\end{aligned}
$$

where

$$
A=-\frac{2 c_{1}+3 c_{2}+c_{3}+c_{4}}{2 c_{123}}, \quad B=-\frac{\left(2-c_{14}\right) c_{-}}{2 c_{1}-c_{1}^{2}+c_{3}^{2}}
$$

[1] C.M. Will, Theory and Experiment in Gravitational Physics, (Cambridge Univ. Press, Cambridge, 1993).

[2] C. M. Will, "The confrontation between general relativity and experiment," Living Rev. Rel. 4, 4 (2001) arXiv:gr-qc/0103036.

[3] C. Eling, T. Jacobson and D. Mattingly, "Einstein-aether theory," arXiv:gr-qc/0410001.

[4] R. M. Wald, General Relativity (University of Chicago Press, 1984).

[5] D. Mattingly, "Modern tests of Lorentz invariance," arXiv:gr-qc/0502097.

[6] R. Bluhm, "Overview of the SME: Implications and phenomenology of Lorentz violation," arXiv:hep-ph/0506054.

[7] S. M. Carroll and E. A. Lim, "Lorentz-violating vector fields slow the universe down," Phys. Rev. D 70, 123525 (2004) arXiv:hep-th/0407149.

[8] C. Eling and T. Jacobson, "Static post-Newtonian equivalence of GR and gravity with a dynamical preferred frame," Phys. Rev. D 69, 064005 (2004) arXiv:gr-qc/0310044. 
[9] M. L. Graesser, A. Jenkins and M. B. Wise, "Spontaneous Lorentz violation and the long-range gravitational preferred-frame effect," Phys. Lett. B 613, 5 (2005) arXiv:hep-th/0501223.

[10] R. W. Hellings and K. Nordtvedt, Jr., "Vector-Metric theory of gravity," Phys. Rev. D 7, 3593 (1973).

[11] D. Mattingly and T. Jacobson, "Relativistic gravity with a dynamical preferred frame," in CPT and Lorentz Symmetry II, ed. V.A. Kostelecky (World Scientific, Singapore, 2002) arXiv:gr-qc/0112012.

[12] E. A. Lim, "Can We See Lorentz-Violating Vector Fields in the CMB?," arXiv:astro-ph/0407437.

[13] J. W. Elliott, G. D. Moore and H. Stoica, "Constraining the new aether: Gravitational Cherenkov radiation," JHEP 0508, 066 (2005) arXiv:hep-ph/0505211.

[14] T. Jacobson and D. Mattingly, "Einstein-Aether waves," Phys. Rev. D 70, 024003 (2004) arXiv:gr-qc/0402005.

[15] C. Eling, "Energy in the Einstein-aether theory," arXiv:gr-qc/0507059.

[16] B. Z. Foster, "Radiation damping in Einstein-aether theory," arXiv:gr-qc/0602004.

[17] C. Eling and T. Jacobson, in preparation. 